\title{
EFFECT OF SEEDING RATE AND NITROGEN FERTILIZATION ON THE YIELD OF FLAX
}

\author{
A.E.A. Omar and Salwa, M.A.I. Ash-Shormillesy
}

Agronomy Department, Faculty of Agriculture, Zagazig University, Zagazig, Egypt.

\section{ABSTRACT}

This field study was performed at the Agricultural Research Farm (Ghazala location), Faculty of Agriculture, Zagazig University., Egypt during 2002/2003 and 2003/2004 seasons to evaluate the effect of seeding rate (expressed herein as seed number, being 800, 1200 and 1600 seeds $\left./ \mathrm{m}^{2}\right)$ and $N$ fertilization levels $(20,40$ and 60 $\mathrm{kgN} / \mathrm{fad}$ ) on the yield and some certain characters of the two flax genotypes (local cultivar Giza 7 and Imported genotype Elise). In each trial, split-split plot design of three replicates was employed, with 2 genotypes as main plots, the three seeding rates as sub-plots and the three $N$ levels as sub-sub plots. The experimental unit was $6 \mathrm{~m}^{2}$ with dimensions of $2 \times 3 \mathrm{~m}$.

The results showed significant cultivar behaviour, since Giza 7 cultivar was markedly distinguished on its similar Elise one in each of : stem diameter, number of basal and apical branches / plant, straw yield/fad, capsule and seed numbers/plant, seed index, seed yield/fad, seed oil percentage and oil yield/fad. On the other hand, Elise genotype was significantly superior as for: plant height, technical stem length, fibre\% and fibre yield/fad. Such trend was greatly valid in both seasons and over them.

The three numbers of seeds $/ \mathrm{m}^{2}$ gave considerable response in all flax traits studied, since planting 800 seeds $/ \mathrm{m}^{2}$ gave greater mean values respecting : stem diameter, number of basal and apical branches/plant, capsule and seed numbers / plant, seed index and seed oil content. Moreover, planting 1600 seeds $/ \mathrm{m}^{2}$ possessed greater excess in each of : plant height, technical stem length, fibre\% and at last the final yields/fad of straw, fibre, seed and oil when compared with both 800 and 1200 seeding rates. This phenomenon was completely true in both trials and across them as well.

Nitrogen fertilization levels exerted noticeable variations in all tested flax traits, since the $20 \mathrm{~kg} \mathrm{~N}$ dose was most effective in raising both fibre\% and seed oil content. Likewise, the $60 \mathrm{~kg} \mathrm{~N}$ level attained the greatest mean records regarding plant height, technical stem length, stem diameter, number of basal and apical branches/plant, straw and fibre yields/fad, capsule and seed numbers / plant, seed index and both seed and oil yields/unit area. Such N levels trend was fairly manifested in both seasons and their pooled data as well.

Planting 1600 seeds $/ \mathrm{m}^{2}$ interacted meaningly with $60 \mathrm{~kg}$ N/fad to produce pronounced increments as for : plant height, fibre\% and fibre yield/fad (respecting Elise genotype), number of basal branches/plant as well as straw, seed and oil yields/fad (in case of Giza 7 cultivar). In other score, the final yields/fad from straw, fibre, seed and oil were markedly maximized by considering the 1600 seeding rate along with $60 \mathrm{~kg} \mathrm{~N}$ level/fad under the circumstances of this investigation.

Key words : Seeding rate, nitrogen fertilization, flax. 


\section{INTRODUCTION}

Flax (Linum usitatissimum L.) is one of the most important fibre and oil crops. In Egypt, such crop orders second after cotton as a fiber crop. Both fibre and oil production of flax are insufficient to face the highly local consumption of the ever growing population. So, the major concern of agronomists and government is to attain the maximum flax yield per unit land area by applying the most suitable cultural practices, such as the proper plant stand density and suitable $\mathrm{N}$ levels which can boost flax productivity, especially for the new released and high yielding genotypes. Many workers evaluated certain flax genotypes behaviour as for different agronomic traits. Ash-Shormillesy (2001) made a comparison among three flax genotypes, being Giza 7, Belinka and Elise and reported that plant height, technical stem length and straw, fibre as well as seed yields/fad were in favour of the Elise genotype. While, Giza $7 \mathrm{cv}$ was significantly distinguished regarding number of basal or apical branches/plant, straw yield/plant or/fad, fruiting zone length, number of capsules and seeds/plan, seed number/capsule, seed yield/plant, seed index and both seed as well as oil yields/fad. At the same-time Belinka genotype had intermediate mean values regarding the above- mentioned characters. Similar cultivar variations respecting most agronomic traits were documented by other investigators, including : El-Sweify et al. (1996), Kineber et al. (1997), El-Sabbagh et al. (1998), El-Gazzar and Abo-Zaied (2001), El-Azzouni et al. (2003) and at last Kineber (2003).

It had long been established that one of the major factors limiting flax yield and its related characters is the suitable plant density / unit area. Sincewhile; Esmail and Morsy (1994) tried the effect of 750,1000, 1250, 1500, 1750 and 2000 seeds $/ \mathrm{m}^{2}$ on some certain criteria of Giza 6 flax cultivar and concluded that planting 750 seeds $/ \mathrm{m}^{2}$ reflected significant increments respecting : number of fruiting branches and capsules/plant, the final yields/plant from seed; straw and fibre as well as seed oil content. Meanwhile, the highest seeding rate of 2000 seeds $/ \mathrm{m}^{2}$ detected its visible effect as for : plant height, technical stem length, fruiting zone length and both fibre and seed yields/fad. The other seeding rates found between 750 and 2000 seeds $/ \mathrm{m}^{2}$ gave intermediate averages respecting the previous characters. Other works documented similar findings on most flax plant characters due to different seeding rates/fad or seed number/ $\mathrm{m}^{2}$, such as : Ash-Shormillesy (2001), El-Gazzar and Abou-Zaied (2001), El-Gazzar (2005) and at last El-Deeb et al. (2006).

Several investigators have examined the positive response of flax plants to different $\mathrm{N}$ levels, of them : Zedan et al. (1997), working on Giza 7 flax cultivar, showed that the $70 \mathrm{~kg}$ $\mathrm{N}$ level/fad detected significant increases as regard : plant height, technical stem length, straw yield either/plant or/fad, fibre yield/fad, upper branching zone length, capsule number/plant, seed number/capsule and/plant and at last seed yield/plant or /fad when compared with the other $\mathrm{N}$ levels tested, being 50 and $60 \mathrm{~kg} \mathrm{~N} / \mathrm{fad}$. Mean-time, seed index was insignificantly affected by the different $\mathrm{N}$ levels tried. Other researchers recorded similar N level differences as for most agronomic flax traits, such as : Ghanem (1990), Kineber et al. (1998), El-Gazar and Abou-Zaied (2001), El-Gazzar and Kineber (2002), Moustafa et al. (2003) and at last El-Gazzar and El-Kady (2005).

Accordingly, this study was done to explore the effect of seeding rate (assembled herein as seed number $/ \mathrm{m}^{2}$ ) and $\mathrm{N}$ fertilization levels on certain important characters of both Giza 7 and Elise genotypes. 


\section{MATERIALS AND METHDOS}

Two field trials were executed during 2002/2003 and 2003/2004 seasons at the Agricultural Research Farm. (Ghazala location), Faculty of Agriculture, Zagazig University, Egypt to investigate the effect of seeding rates and $\mathrm{N}$ fertilization levels on straw, fibre, seed and oil yields beside their related characters of both Giza 7 and Elise flax cultivars. The soil of the experiments was clay in texture with $\mathrm{pH}$ value of $7.8,1.35$ organic matter and having 20, 17 and $220 \mathrm{ppm}$ available $\mathrm{N}, \mathrm{P}$ and $\mathrm{K}$, successively (averages of both seasons for the upper $30 \mathrm{~cm}$ of soil).

\section{The studied factors :}

The experimental factors examined were as below :

I- Cultivars; V : Two flax cultivars evaluated were :

1- Giza 7, local and commercial cultivar (dual purpose).

2- Elise genotype, introduced from Holland in 1996, considered as fibre type.

II. Seeding rates, $\mathbf{S}$ : Three seeding rates expressed as seed number $/ \mathrm{m}^{2}$ which were :
$1-800$
2- 1200
3- 1600

Each seeding rate used was computed as $\mathrm{kg}$ seeds/fad on the basic weight of 1000seed weight for each cultivar according to the recommended rate described by El-Gazzar (1990) on similar flax cultivars (Table 1).

III- Nitrogen fertilization, $\mathbf{N}$ : Three $\mathrm{N}$ levels tried in this study were as following :

$$
\begin{array}{lll}
1-20 \mathrm{~kg} \mathrm{~N} / \mathrm{fad} \text {. } & 2-40 \mathrm{~kg} \mathrm{~N} / \mathrm{fad} . & 3-60 \mathrm{~kg} \mathrm{~N} / \mathrm{fad} \text {. }
\end{array}
$$

\section{Design of the field trials :}

The experimental design used was split-split plot with three replicates. The main plots were occupied by the two flax cultivars, and the three seeding rates were randomly allocated in the sub-plots. Whereas, the sub-sub plots were devoted to the three $\mathrm{N}$ levels. The size of each experimental unit was $6 \mathrm{~m}^{2}$ in both trials, having ten rows (drills) of $3 \mathrm{~m}$ in length and $20 \mathrm{~cm}$ in width.

Table 1. Seeding rate (kg seeds/fad) assembled as seed number $/ \mathrm{m}^{2}$ based on 1000 -seed weight (8.5 or $6.3 \mathrm{gm}$ of both Giza 7 and Elise genotypes).

\begin{tabular}{ccc}
\hline \multirow{2}{*}{ Seed number $/ \mathbf{m}^{2}$} & \multicolumn{2}{c}{ Seeding rate as kg seeds/fad. } \\
\cline { 2 - 3 } & Giza 7 cultivar & Elise genotype \\
\hline 800 & 28.56 & 21.17 \\
1200 & 42.84 & 31.75 \\
1600 & 57.12 & 42.34 \\
\hline
\end{tabular}

\section{Cultural practices :}

The preceding crop was maize in both seasons. After seed-bed preparation, all experimental plots were fertilized with $15.5 \mathrm{~kg} \mathrm{P}_{2} \mathrm{O}_{5}$ and $25.0 \mathrm{~kg} \mathrm{~K}_{2} \mathrm{O} /$ fad in the form of calcium super-phosphate $\left(15.5 \% \mathrm{P}_{2} \mathrm{O}_{5}\right)$ and potassium sulphate $\left(48-52 \% \mathrm{~K}_{2} \mathrm{O}\right)$, respectively. The seeds of both Giza 7 and Elise cultivars were mixed well with the recommended fungicide to control both seed and seedling diseases. Seeding rates used (expressed as seed 
number $/ \mathrm{m}^{2}$ ) differed between the two tested genotypes due to varying the seed index of each cultivar as shown in Table 1. The seeds of both genotypes were sown by using Afir method in drills of $20 \mathrm{~cm}$ apart on November 28 and 20 in first and second seasons, orderly. Nitrogen fertilizer levels of 20, 40 and $60 \mathrm{~kg} \mathrm{~N} / \mathrm{fad}$ as per treatment were added in form of urea fertilizer $(46.5 \% \mathrm{~N})$ in two equal portions before the first and second irrigations, successively. All other practices were manually adopted on proper time and usually applied as recommended in flax production. At last, harvesting was done on May $21^{\text {st }}$ and $30^{\text {th }}$ in the two following seasons, respectively.

\section{The studied characters :}

At harvest, ten guarded plants from the third drill in each experimental unit of the 3 replicates were taken to record the following traits :

1- Plant height $(\mathrm{cm})$ 2- Technical stem length (effective length), $\mathrm{cm}$.

3- Stem diameter $(\mathrm{mm})$. 4- Number of basal branches/plant.

5- Number of apical branches/plant.

Then, the plants located in one $\mathrm{m}^{2}$ of each sub-sub plot were uprooted to estimate :

6- Straw yield/fad (ton).

Fibre yield /fad.

7- Fibre percentage $=\frac{}{\text { The retted straw yield/fad. }} \quad$ x 100

8- Fibre yield/fad (ton).

To determine fibre yield/fad, the plants were weighed (after seed separation) and pooled in water of cement basins to be naturally retted. Then, the plants were left to be dried naturally and the flax fibres were mechanically separated to record fibre yield as $\mathrm{kg}$ on plot area basis, then converted to ton/fad.

9- Capsule number/plant. 10- Seed number/plant.

11- Seed index (1000-seed weight), gm.

All 9, 10 and 11 traits were recorded from the same ten plants that used in the determination of $1-5$ characters.

12- Seed yield/fad (ton). Recorded on plot area basis (from $1 \mathrm{~m}^{2}$ ).

13- Seed oil content (percentage). Estimated by following the method described by A.O.A.C. (1980).

14- Oil yield/fad (ton). It was calculated by multiplying the seed yield/fad (ton) by its seed oil content and dividing by 100 .

\section{Statistical analysis :}

The collected data of both seasons were statistically analyzed by using the split-split plot design procedure as described by Das and Giri (1986). Withal, the combined analysis of variance was also computed for all traits recorded herein, after establishing by Barlett's homogeneity test, where the error variance of the individual season was homogeneous. The significant differences among treatment means were judged with the help of Duncan's multiple range test (Duncan, 1955). In interaction Tables recorded on pooled data basis, capital and small letters were used to compare both row and column means, successively. $*$, ** and N.S. are symbols found in all listed Tables to verify the significant differences 
among treatment means at 5 and $1 \%$ levels of probability and insignificant differences, orderly.

\section{RESULTS AND DISCUSSION}

\section{I- Cultivar behaviour :}

Significant cultivar differences are shown in all the studied characters of flax in both seasons and over them, since the local cultivar Giza 7 gave greater mean averages than the introduced Elise one as for : stem diameter, number of basal and apical branches/plant, straw yield/fad, capsule and seed numbers/plant, seed index, seed yield/fad, seed oil content and the final oil yield/fad. On the other hand, the Elise genotype was superior to Giza 7 one in each of : plant height, technical stem length, fibre percentage and fibre yield per unit land area. Such trend was, also seen in both trials and in their pooled data as well (Tables 2 to 6, orderly). The cultivar differences in the recorded traits may be ascribed to the genetical make up and the response of each genotype to the prevailing environmental conditions found in the surrounding media. Similar flax cultivar differences in most agronomic characters were cited by other investigators, of them : El-Swiefy et al. (1996), Kineber et al. (1997), El-Sabbagh et al. (1998), El-Gazzar and Abou-Zaied (2001), AshShormillesy (2001), El-Azzouni et al. (2003) and Kineber (2003).

\section{II- Seeding rates effect :}

The three seeding rates as number of seeds $/ \mathrm{m}^{2}$ varied considerably in their mean values in all the tested flax traits in both seasons and across them, where the light density of 800 seeds $/ \mathrm{m}^{2}$ detected greater mean averages regarding : stem diameter, number of basal and apical branches/plant, capsule as well as seed numbers/plant, 1000-seed weight and at last seed oil content. The increase in the afore-named characters due to the $800 \mathrm{seeds} / \mathrm{m}^{2}$ might be attributed to the wide open canopy structure that allowed better light and air penetration and, therefore increased the metabolism in flax plant tissues. Furthermore, the dense density of 1600 seeds $/ \mathrm{m}^{2}$ reflected the greatest mean records as for : plant height, technical stem length, straw yield/fad, fibre percentage and the final yields/fad from fibre, seed and oil. In this regard, the crowded plants established from 1600 seeds $/ \mathrm{m}^{2}$ could improve the canopy architecture efficiency, leading to marked increases in final flax yields per unit land area as shown previously. At the same-time, the second plant stand of 1200 seeds $/ \mathrm{m}^{2}$ gave medium mean averages in all tested flax traits in both trials and over them as demonstrated in Tables $2-6$, successively. The effect of seeding rates or seed numbers on most agronomic traits of flax was documented by several workers, among them : Esmail and Morsy (1994), El-Gazzar and Abou-Zaied (2001), El-Gazzar (2005) and El-Deeb et al. (2006). 
Table 2 : Plant height $(\mathrm{cm})$, technical stem length $(\mathrm{cm})$ and stem diameter $(\mathrm{mm})$ of flax due to various treatments during 2002/2003 and 2003/2004 seasons.

\begin{tabular}{|c|c|c|c|c|c|c|c|c|c|}
\hline \multirow{2}{*}{ Treatments } & \multicolumn{3}{|c|}{$\begin{array}{l}\text { Plant height } \\
(\mathrm{cm})\end{array}$} & \multicolumn{3}{|c|}{$\begin{array}{c}\text { Technical stem length } \\
(\mathrm{cm})\end{array}$} & \multicolumn{3}{|c|}{$\begin{array}{c}\text { Stem diameter } \\
(\mathbf{m m})\end{array}$} \\
\hline & $\begin{array}{c}\text { First } \\
\text { season }\end{array}$ & $\begin{array}{c}\text { Second } \\
\text { season }\end{array}$ & Comb. & $\begin{array}{c}\text { First } \\
\text { season }\end{array}$ & $\begin{array}{c}\text { Second } \\
\text { season }\end{array}$ & Comb. & $\begin{array}{c}\text { First } \\
\text { season } \\
\end{array}$ & $\begin{array}{l}\text { Second } \\
\text { season }\end{array}$ & Comb. \\
\hline \multicolumn{10}{|l|}{ Cultivars, V: } \\
\hline Giza 7 & $85.56 \mathrm{~b}$ & $93.48 b$ & $89.52 b$ & $70.45 b$ & $77.65 b$ & $74.05 b$ & $2.10 \mathrm{a}$ & $2.14 \mathrm{a}$ & $2.12 \mathrm{a}$ \\
\hline Elise & $94.96 a$ & $95.60 \mathrm{a}$ & $95.28 \mathrm{a}$ & $78.57 \mathrm{a}$ & $82.53 a$ & $80.55 a$ & $1.96 b$ & $2.00 \mathrm{~b}$ & $1.98 \mathrm{~b}$ \\
\hline F. test & $* *$ & * & $* *$ & $* *$ & $* *$ & $* *$ & $*$ & * & $*$ \\
\hline \multicolumn{10}{|c|}{ Seed number $/ \mathrm{m}^{2}, \mathrm{~S}:$} \\
\hline 800 & $82.42 \mathrm{c}$ & $92.14 \mathrm{c}$ & $87.28 \mathrm{c}$ & $71.84 \mathrm{c}$ & $77.74 \mathrm{c}$ & $74.79 \mathrm{c}$ & $2.26 \mathrm{a}$ & $2.28 \mathrm{a}$ & $2.27 \mathrm{a}$ \\
\hline 1200 & $90.25 b$ & $94.75 b$ & $92.50 \mathrm{~b}$ & $75.53 b$ & $80.03 b$ & $77.78 \mathrm{~b}$ & $1.95 \mathrm{~b}$ & $2.01 \mathrm{~b}$ & $1.98 \mathrm{~b}$ \\
\hline 1600 & $98.11 \mathrm{a}$ & $96.73 a$ & $97.42 \mathrm{a}$ & $76.16 a$ & $82.50 \mathrm{a}$ & $79.33 \mathrm{a}$ & $1.88 \mathrm{c}$ & $1.92 \mathrm{c}$ & $1.90 \mathrm{c}$ \\
\hline F. test & $* *$ & $* *$ & $* *$ & $*$ & $* *$ & $* *$ & $* *$ & $* *$ & $*$ \\
\hline \multicolumn{10}{|c|}{$\mathrm{N}$ levels (kgN/fad), $\mathrm{N}$ : } \\
\hline 20 & $86.06 \mathrm{c}$ & $91.78 \mathrm{c}$ & $88.92 \mathrm{c}$ & $71.59 \mathrm{c}$ & $76.85 \mathrm{c}$ & $74.22 \mathrm{c}$ & $1.88 \mathrm{c}$ & $1.90 \mathrm{c}$ & $1.89 \mathrm{c}$ \\
\hline 40 & $91.34 \mathrm{~b}$ & $94.10 \mathrm{~b}$ & $92.72 b$ & $74.79 b$ & $80.99 b$ & $77.89 \mathrm{~b}$ & $2.04 \mathrm{~b}$ & $2.08 \mathrm{~b}$ & $2.06 \mathrm{~b}$ \\
\hline 60 & $93.38 \mathrm{a}$ & $97.74 a$ & $95.56 a$ & $77.15 \mathrm{a}$ & $82.43 a$ & $79.79 a$ & $2.17 \mathrm{a}$ & $2.23 \mathrm{a}$ & $2.20 \mathrm{a}$ \\
\hline F. test & $* *$ & ** & $* *$ & ** & $* *$ & ** & $* *$ & $* *$ & $* *$ \\
\hline \multicolumn{10}{|l|}{ Interactions : } \\
\hline $\mathrm{V} \times \mathrm{S}$ & $* *$ & N.S & ** & ** & N.S & N.S & N.S & N.S & N.S \\
\hline $\mathrm{V} \times \mathrm{N}$ & $*$ & N.S & $*$ & N.S & N.S & N.S & N.S & N.S & N.S \\
\hline $\mathrm{S} \times \mathrm{N}$ & N.S & N.S & N.S & N.S & N.S & N.S & $*$ & N.S & N.S \\
\hline
\end{tabular}

Table 3 : Number of basal and apical branches/plant as well as straw yield/fad (ton) of flax due to various treatments during $2002 / 2003$ and 2003/2004 seasons.

\begin{tabular}{|c|c|c|c|c|c|c|c|c|c|}
\hline \multirow{2}{*}{ Treatments } & \multicolumn{3}{|c|}{$\begin{array}{l}\text { Number of basal } \\
\text { branches/plant }\end{array}$} & \multicolumn{3}{|c|}{$\begin{array}{c}\text { Number of apical } \\
\text { branches/plant }\end{array}$} & \multicolumn{3}{|c|}{$\begin{array}{c}\text { Straw yield/fad } \\
\text { (ton) }\end{array}$} \\
\hline & $\begin{array}{c}\text { First } \\
\text { season }\end{array}$ & $\begin{array}{l}\text { Second } \\
\text { season }\end{array}$ & Comb. & $\begin{array}{l}\text { First } \\
\text { season }\end{array}$ & $\begin{array}{l}\text { Second } \\
\text { season }\end{array}$ & Comb. & $\begin{array}{c}\text { First } \\
\text { season }\end{array}$ & $\begin{array}{l}\begin{array}{l}\text { Second } \\
\text { season }\end{array} \\
\end{array}$ & Comb. \\
\hline \multicolumn{10}{|l|}{ Cultivars, V : } \\
\hline Giza 7 & $1.32 \mathrm{a}$ & $1.60 \mathrm{a}$ & $1.46 \mathrm{a}$ & $3.75 \mathrm{a}$ & $4.67 \mathrm{a}$ & $4.21 \mathrm{a}$ & $3.56 \mathrm{a}$ & $3.84 \mathrm{a}$ & $3.70 \mathrm{a}$ \\
\hline Elise & $1.02 \mathrm{~b}$ & $1.14 b$ & $1.08 \mathrm{~b}$ & $3.31 \mathrm{~b}$ & $4.11 b$ & $3.71 b$ & $2.68 \mathrm{~b}$ & $2.72 b$ & $2.70 \mathrm{~b}$ \\
\hline F. test & $* *$ & $*$ & $* *$ & $*$ & $*$ & $*$ & $*$ & $*$ & $*$ \\
\hline \multicolumn{10}{|l|}{ Seed number $/ \mathrm{m}^{2}, \mathrm{~S}$ : } \\
\hline 800 & $1.33 \mathrm{a}$ & $1.63 \mathrm{a}$ & $1.48 \mathrm{a}$ & $4.06 \mathrm{a}$ & $4.62 \mathrm{a}$ & $4.34 \mathrm{a}$ & $2.90 \mathrm{c}$ & $2.48 \mathrm{c}$ & $2.69 \mathrm{c}$ \\
\hline 1200 & $1.18 \mathrm{~b}$ & $1.40 \mathrm{~b}$ & $1.29 b$ & $3.45 b$ & $4.53 b$ & $3.99 \mathrm{~b}$ & $2.46 \mathrm{~b}$ & $3.40 \mathrm{~b}$ & $2.93 b$ \\
\hline 1600 & $1.00 \mathrm{c}$ & $1.08 \mathrm{c}$ & $1.04 \mathrm{c}$ & $3.08 \mathrm{c}$ & $4.02 \mathrm{c}$ & $3.55 \mathrm{c}$ & $4.00 \mathrm{a}$ & $3.96 \mathrm{a}$ & $3.98 \mathrm{a}$ \\
\hline F. test & $* *$ & $* *$ & $* *$ & $* *$ & $*$ & $* *$ & $* *$ & $* *$ & $* *$ \\
\hline \multicolumn{10}{|l|}{$\mathrm{N}$ levels (kgN/fad), $\mathrm{N}$ : } \\
\hline 20 & $0.85 \mathrm{c}$ & $1.11 \mathrm{c}$ & $0.98 \mathrm{c}$ & $3.17 \mathrm{c}$ & $3.87 \mathrm{c}$ & $3.52 \mathrm{c}$ & $2.51 \mathrm{c}$ & $2.63 c$ & $2.57 \mathrm{c}$ \\
\hline 40 & $1.17 \mathrm{~b}$ & $1.33 \mathrm{~b}$ & $1.25 \mathrm{~b}$ & $3.56 \mathrm{~b}$ & $4.44 b$ & $4.00 \mathrm{~b}$ & $3.02 \mathrm{~b}$ & $3.18 \mathrm{~b}$ & $3.10 \mathrm{~b}$ \\
\hline 60 & $1.49 \mathrm{a}$ & $1.67 \mathrm{a}$ & $1.58 \mathrm{a}$ & $3.86 \mathrm{a}$ & $4.86 \mathrm{a}$ & $4.36 \mathrm{a}$ & $3.83 \mathrm{a}$ & $4.03 \mathrm{a}$ & $3.93 \mathrm{a}$ \\
\hline F. test & $* *$ & $* *$ & $* *$ & $* *$ & $* *$ & $* *$ & $* *$ & $* *$ & $* *$ \\
\hline \multicolumn{10}{|l|}{ Interactions : } \\
\hline $\mathrm{V} \times \mathrm{S}$ & N.S & N.S & N.S & N.S & N.S & N.S & N.S & $*$ & $*$ \\
\hline $\mathrm{V} \times \mathrm{N}$ & N.S & $* *$ & $*$ & N.S & N.S & N.S & $*$ & $*$ & $* *$ \\
\hline $\mathrm{S} \times \mathrm{N}$ & N.S & N.S & N.S & N.S & N.S & N.S & $*$ & $*$ & $* *$ \\
\hline
\end{tabular}


Table 4 : Fibre percentage, fibre yield/fad (ton) and number of capsules/plant of flax as affected by the different treatments during 2002/2003 and 2003/2004 seasons.

\begin{tabular}{|c|c|c|c|c|c|c|c|c|c|}
\hline \multirow{2}{*}{ Treatments } & \multicolumn{3}{|c|}{ Fibre\% } & \multicolumn{3}{|c|}{$\begin{array}{l}\text { Fibre yield/fad } \\
\text { (ton) }\end{array}$} & \multicolumn{3}{|c|}{ Capsule number/plant } \\
\hline & $\begin{array}{c}\text { First } \\
\text { season }\end{array}$ & $\begin{array}{l}\text { Second } \\
\text { season }\end{array}$ & Comb. & $\begin{array}{c}\text { First } \\
\text { season }\end{array}$ & $\begin{array}{l}\text { Second } \\
\text { season }\end{array}$ & Comb. & $\begin{array}{c}\text { First } \\
\text { season }\end{array}$ & $\begin{array}{l}\text { Second } \\
\text { season }\end{array}$ & Comb. \\
\hline \multicolumn{10}{|l|}{ Cultivars, V: } \\
\hline Giza 7 & $19.15 b$ & $21.37 \mathrm{~b}$ & $20.26 b$ & $0.448 b$ & $0.480 \mathrm{~b}$ & $0.464 b$ & $14.62 \mathrm{a}$ & $19.24 \mathrm{a}$ & $16.93 \mathrm{a}$ \\
\hline Elise & $27.37 \mathrm{a}$ & $27.79 a$ & $27.58 \mathrm{a}$ & $0.548 \mathrm{a}$ & $0.552 \mathrm{a}$ & $0.550 \mathrm{a}$ & $12.80 \mathrm{~b}$ & $15.46 b$ & $14.13 b$ \\
\hline F. test & $* *$ & $* *$ & $* *$ & $*$ & $*$ & $*$ & $*$ & $*$ & $*$ \\
\hline \multicolumn{10}{|l|}{ Seed number $/ \mathrm{m}^{2}, \mathrm{~S}$ : } \\
\hline 800 & $21.14 \mathrm{c}$ & $22.80 \mathrm{c}$ & $21.97 \mathrm{c}$ & $0.486 \mathrm{c}$ & $0.494 \mathrm{c}$ & $0.490 \mathrm{c}$ & $16.73 a$ & $18.99 \mathrm{a}$ & $17.86 \mathrm{a}$ \\
\hline 1200 & $22.76 b$ & $24.26 b$ & $23.51 b$ & $0.495 b$ & $0.519 b$ & $0.507 \mathrm{~b}$ & $12.94 b$ & $17.64 b$ & $15.29 b$ \\
\hline 1600 & $25.88 \mathrm{a}$ & $26.68 \mathrm{a}$ & $26.28 \mathrm{a}$ & $0.513 \mathrm{a}$ & $0.535 \mathrm{a}$ & $0.524 \mathrm{a}$ & $11.46 \mathrm{c}$ & $15.42 \mathrm{c}$ & $13.44 \mathrm{c}$ \\
\hline F. test & $* *$ & $* *$ & $* *$ & $* *$ & $* *$ & $* *$ & $* *$ & $* *$ & $* *$ \\
\hline \multicolumn{10}{|c|}{ N levels $(\mathrm{kgN} / \mathrm{fad}), \mathbf{N}$ : } \\
\hline 20 & $28.21 \mathrm{a}$ & $28.57 \mathrm{a}$ & $28.39 \mathrm{a}$ & $0.476 \mathrm{c}$ & $0.484 \mathrm{c}$ & $0.480 \mathrm{c}$ & $11.84 \mathrm{c}$ & $15.24 \mathrm{c}$ & $13.54 \mathrm{c}$ \\
\hline 40 & $22.99 b$ & $24.67 b$ & $23.83 b$ & $0.497 \mathrm{~b}$ & $0.517 b$ & $0.507 b$ & $13.66 \mathrm{~b}$ & $17.64 b$ & $15.65 b$ \\
\hline 60 & $18.58 \mathrm{c}$ & $20.50 \mathrm{c}$ & $19.54 \mathrm{c}$ & $0.521 \mathrm{a}$ & $0.547 \mathrm{a}$ & $0.534 \mathrm{a}$ & $15.63 a$ & $19.17 \mathrm{a}$ & $17.40 \mathrm{a}$ \\
\hline F. test & $* *$ & $* *$ & $* *$ & $* *$ & $* *$ & $* *$ & $* *$ & $* *$ & $* *$ \\
\hline \multicolumn{10}{|l|}{ Interactions : } \\
\hline $\mathrm{V} \times \mathrm{S}$ & N.S & N.S & $*$ & $* *$ & $*$ & $*$ & $* *$ & N.S & N.S \\
\hline $\mathrm{V} \times \mathrm{N}$ & N.S & N.S & N.S & $*$ & $*$ & $*$ & N.S & N.S & N.S \\
\hline $\mathrm{S} \times \mathrm{N}$ & N.S & N.S & N.S & $*$ & N.S & $*$ & N.S & N.S & N.S \\
\hline
\end{tabular}

Table 5 : Seed number/plant, seed index (gm) and seed yield/fad (ton) of flax in response to the different treatments in 2002/2003 and 2003/2004 seasons.

\begin{tabular}{|c|c|c|c|c|c|c|c|c|c|}
\hline \multirow{2}{*}{ Treatments } & \multicolumn{3}{|c|}{$\begin{array}{c}\text { Seed number/ } \\
\text { plant }\end{array}$} & \multicolumn{3}{|c|}{$\begin{array}{l}\text { 1000-seed weight (seed } \\
\text { index), gm }\end{array}$} & \multicolumn{3}{|c|}{$\begin{array}{l}\text { Seed yield/fad } \\
\text { (ton) }\end{array}$} \\
\hline & $\begin{array}{c}\text { First } \\
\text { season }\end{array}$ & $\begin{array}{l}\text { Second } \\
\text { season }\end{array}$ & Comb. & $\begin{array}{c}\text { First } \\
\text { season }\end{array}$ & $\begin{array}{l}\text { Second } \\
\text { season }\end{array}$ & Comb. & $\begin{array}{c}\text { First } \\
\text { season }\end{array}$ & $\begin{array}{l}\text { Second } \\
\text { season }\end{array}$ & Comb. \\
\hline \multicolumn{10}{|l|}{ Cultivars, V: } \\
\hline Giza 7 & $102.34 \mathrm{a}$ & $130.00 \mathrm{a}$ & $116.17 \mathrm{a}$ & $8.58 \mathrm{a}$ & $8.62 \mathrm{a}$ & $8.60 \mathrm{a}$ & $0.906 a$ & $0.952 \mathrm{a}$ & $0.929 a$ \\
\hline Elise & $76.82 b$ & $92.00 \mathrm{~b}$ & $84.41 b$ & $6.44 b$ & $7.08 \mathrm{~b}$ & $6.76 b$ & $0.802 \mathrm{~b}$ & $0.816 b$ & $0.809 b$ \\
\hline F. test & $* *$ & $* *$ & $* *$ & $* *$ & $*$ & $*$ & $*$ & $*$ & $*$ \\
\hline \multicolumn{10}{|l|}{ Seed number $/ \mathrm{m}^{2}, \mathrm{~S}$ : } \\
\hline 800 & $114.10 \mathrm{a}$ & $126.00 \mathrm{a}$ & $120.05 a$ & $8.54 \mathrm{a}$ & $9.00 \mathrm{a}$ & $8.77 \mathrm{a}$ & $0.800 \mathrm{c}$ & $0.814 \mathrm{c}$ & $0.807 \mathrm{c}$ \\
\hline 1200 & $81.94 b$ & $114.50 \mathrm{~b}$ & $98.22 b$ & $7.50 \mathrm{~b}$ & $7.92 b$ & $7.71 b$ & $0.858 \mathrm{~b}$ & $0.864 b$ & $0.861 b$ \\
\hline 1600 & $72.70 \mathrm{c}$ & $92.50 \mathrm{c}$ & $82.60 \mathrm{c}$ & $6.49 \mathrm{c}$ & $6.63 c$ & $6.56 c$ & $0.904 a$ & $0.974 \mathrm{a}$ & $0.939 \mathrm{a}$ \\
\hline F. test & $* *$ & $* *$ & $* *$ & $* *$ & $* *$ & $* *$ & $* *$ & $* *$ & $* *$ \\
\hline \multicolumn{10}{|l|}{ N levels (kgN/fad), $\mathbf{N}:$} \\
\hline 20 & $73.74 \mathrm{c}$ & $90.00 \mathrm{c}$ & $81.87 \mathrm{c}$ & $6.20 \mathrm{c}$ & $7.04 \mathrm{c}$ & $6.62 c$ & $0.670 \mathrm{c}$ & $0.704 \mathrm{c}$ & $0.687 \mathrm{c}$ \\
\hline 40 & $85.00 \mathrm{~b}$ & $114.00 \mathrm{~b}$ & $99.50 b$ & $7.71 b$ & $7.93 b$ & $7.82 b$ & $0.868 \mathrm{~b}$ & $0.916 b$ & $0.892 b$ \\
\hline 60 & $110.00 \mathrm{a}$ & $129.00 \mathrm{a}$ & $119.50 \mathrm{a}$ & $8.62 \mathrm{a}$ & $8.58 \mathrm{a}$ & $8.60 \mathrm{a}$ & $1.024 \mathrm{a}$ & $1.032 \mathrm{a}$ & $1.028 \mathrm{a}$ \\
\hline F. test & $* *$ & $* *$ & $* *$ & $* *$ & $* *$ & $* *$ & $* *$ & $* *$ & $* *$ \\
\hline \multicolumn{10}{|l|}{ Interactions : } \\
\hline $\mathrm{V} \times \mathrm{S}$ & N.S & N.S & N.S & $* *$ & $*$ & $*$ & $* *$ & $*$ & $*$ \\
\hline $\mathrm{V} \times \mathrm{N}$ & N.S & $*$ & N.S & N.S & N.S & N.S & $*$ & $* *$ & $*$ \\
\hline $\mathrm{S} \times \mathrm{N}$ & N.S & N.S & N.S & $*$ & $*$ & $*$ & $*$ & $*$ & $* *$ \\
\hline
\end{tabular}


Table 6 : Seed oil content (\%) and oil yield/fad (ton) of flax in response to the different treatments during 2002/2003 and 2003/2004 seasons.

\begin{tabular}{|c|c|c|c|c|c|c|}
\hline \multirow{2}{*}{ Treatments } & \multicolumn{3}{|c|}{$\begin{array}{c}\text { Seed oil content } \\
(\%)\end{array}$} & \multicolumn{3}{|c|}{$\begin{array}{l}\text { Oil yield/fad } \\
\text { (ton) }\end{array}$} \\
\hline & $\begin{array}{c}\text { First } \\
\text { season }\end{array}$ & $\begin{array}{l}\text { Second } \\
\text { season }\end{array}$ & Comb. & $\begin{array}{c}\text { First } \\
\text { season }\end{array}$ & $\begin{array}{c}\text { Second } \\
\text { season }\end{array}$ & Comb. \\
\hline \multicolumn{7}{|l|}{ Cultivars, V : } \\
\hline Giza 7 & $34.96 \mathrm{a}$ & $35.26 \mathrm{a}$ & $35.11 \mathrm{a}$ & $0.316 \mathrm{a}$ & $0.336 \mathrm{a}$ & $0.326 \mathrm{a}$ \\
\hline Elise & $33.74 b$ & $33.92 b$ & $33.83 b$ & $0.270 \mathrm{~b}$ & $0.278 b$ & $0.274 b$ \\
\hline F. test & $*$ & $* *$ & $* *$ & $* *$ & $* *$ & $* *$ \\
\hline \multicolumn{7}{|l|}{ Seed number $/ \mathrm{m}^{2}, \mathrm{~S}$ : } \\
\hline 800 & $35.76 a$ & $35.62 \mathrm{a}$ & $35.69 \mathrm{a}$ & $0.286 \mathrm{c}$ & $0.292 b$ & $0.289 \mathrm{c}$ \\
\hline 1200 & $34.35 b$ & $34.59 \mathrm{~b}$ & $34.47 b$ & $0.293 b$ & $0.301 b$ & $0.297 b$ \\
\hline 1600 & $32.94 \mathrm{c}$ & $33.56 \mathrm{c}$ & $33.25 \mathrm{c}$ & $0.300 \mathrm{a}$ & $0.328 \mathrm{a}$ & $0.314 \mathrm{a}$ \\
\hline F. test & $* *$ & $* *$ & $* *$ & $*$ & $*$ & $* *$ \\
\hline \multicolumn{7}{|l|}{ N levels (kgN/fad), $N$ : } \\
\hline 20 & $35.96 a$ & $35.58 \mathrm{a}$ & $35.77 \mathrm{a}$ & $0.242 \mathrm{c}$ & $0.254 \mathrm{c}$ & $0.248 \mathrm{c}$ \\
\hline 40 & $34.33 b$ & $34.65 b$ & $34.49 b$ & $0.301 b$ & $0.319 b$ & $0.310 \mathrm{~b}$ \\
\hline 60 & $32.76 \mathrm{c}$ & $33.54 \mathrm{c}$ & $33.15 \mathrm{c}$ & $0.336 \mathrm{a}$ & $0.348 \mathrm{a}$ & $0.342 \mathrm{a}$ \\
\hline F. test & $* *$ & $* *$ & $* *$ & $* *$ & $* *$ & $* *$ \\
\hline \multicolumn{7}{|l|}{ Interactions : } \\
\hline $\mathrm{V} \times \mathrm{S}$ & N.S & N.S & N.S & $*$ & $*$ & $*$ \\
\hline $\mathrm{V} \times \mathrm{N}$ & N.S & N.S & N.S & N.S & $*$ & $*$ \\
\hline $\mathrm{S} \times \mathrm{N}$ & N.S & N.S & N.S & $*$ & N.S & $* *$ \\
\hline
\end{tabular}

\section{III- Nitrogen fertilization effect :}

Nitrogen fertilization levels recorded herein excerted significant changes as for all flax characters listed in Tables 2 to 6 , since the highest $\mathrm{N}$ level of $60 \mathrm{~kg} N / f a d$ reflected its considerable excess in each of : plant height, technical stem length, stem diameter, number of basal and apical branches/plant, both straw and fibre yields/fad, capsule and seed numbers/plant, seed index and at last both seed and oil yields per unit area .Upon the pooled data ,the fibre, seed and oil yield increases, due to $60 \mathrm{~kg} \mathrm{~N}$ level/fad reached 11.25 and 5.32; 49.63 and 15.24 and at last 37.90 and $10.32 \%$ over 20 and $40 \mathrm{~kg} \mathrm{~N}$ levels/fad, successively. The reverse hold true respecting both fibre and seed oil percentages, where the highest mean values were in favour of the lowest $\mathrm{N}$ level of $20 \mathrm{~kg} \mathrm{~N} / \mathrm{fad}$. Mean-time, the medium $\mathrm{N}$ rate of $40 \mathrm{~kg} / \mathrm{fad}$ possessed mean averages of the previous characters came in between. The positive effects of the three $\mathrm{N}$ levels tested in this study were shown in both trials and when their combined data were statistically analyzed. The stimulative effect of $\mathrm{N}$ levels added to flax plants of both Giza 7 and Elise genotypes especially in poorly fertility soil like used herein, may be attributed to the stimulating of photosynthesis process, and therefore they encouraged the amounts of metabolites to be accumulated in plant tissues that are responsible for raising the capacity of the fertilized plants to produce more fibre units as well as fruited organs and the consequent fibre and oil yields per unit land area. The vital function of $\mathrm{N}$ nutrition in increasing the final fibre and oil yields/fad as well as the related criteria were documented by other workers, such as : Ghanem (1990), Zedan et al. (1997), Kineber et al. (1998), El-Gazzar and Abou-Zaied (2001), El-Gazzar and Kineber (2002) and Moustafa et al. (2003). 


\section{IV- Interaction effect :}

Data recorded in Table 7 indicate that the interactions between both Giza 7 and Elise genotypes and the three seeding rates of 800,1200 and 1600 seeds $/ \mathrm{m}^{2}$ were significant as for plant height, straw yield/fad, fibre\%, fibre yield/fad, seed index and seed as well as oil yields/fad. The Elise plants had greater value of plant height, fibre $\%$ and fibre yield/fad when were sown at 1600 seeds $/ \mathrm{m}^{2}$. On other score, the Giza 7 plants established at 1600 seeds $/ \mathrm{m}^{2}$ gave the highest records of straw, seed and oil yields per fad. At the same-time, the Giza 7 plants attained the highest value of seed index when 800 seeds $/ \mathrm{m}^{2}$ was applied (Table 7). Intermediate mean averages of the tested traits of Table 7 were recorded due to sowing both Giza 7 and Elise genotypes seeds with 1200 seeds $/ \mathrm{m}^{2}$.

Over the pooled data of both seasons, the $\mathrm{V} \times \mathrm{N}$ interaction gave significant effects on the flax characters of Table 8 indicating the superiority of Elise genotype in plant height and fibre yield/fad due to $60 \mathrm{~kg} \mathrm{~N}$ level. At the other extreme, the flax plants of Giza 7 cultivar got greater averages as for number of basal branches /plant as well as straw, seed and oil yields/fad in response to the highest $\mathrm{N}$ level of $60 \mathrm{~kg} \mathrm{~N} / \mathrm{fad}$. On the other hand, the lowest and medium averages of the tested characters listed in Table 8 were seen in case of 20 or 40 $\mathrm{kg} \mathrm{N}$ levels/fad.

Best of all, the flax plants of 800 seeds $/ \mathrm{m}^{2}$ had greater seed index under $60 \mathrm{~kg} \mathrm{~N}$ level. Moreover, the dense sown plants of $1600 \mathrm{seeds} / \mathrm{m}^{2}$ reflected the greatest mean averages as for the final yields/fad from straw, fibre, seed and oil when the $60 \mathrm{~kg} \mathrm{~N}$ level was concerned (Table 9). Mean-time, the plants of 1200 seeds $/ \mathrm{m}^{2}$ possessed intermediate averages respecting all traits of Table 9 under the three examined $\mathrm{N}$ levels of 20,40 and $60 \mathrm{~kg} / \mathrm{fad}$.

Conclusively, both dual purpose Giza 7 cultivar or fibre genotype Elise were considerably recommended to be grown under Zagazig conditions, Sharkia Governorate, Egypt due to their superior positive response to the dense planting of $1600 \mathrm{seed} / \mathrm{m}^{2}(57.12$ or $42.34 \mathrm{~kg}$ seeds/fad as for Giza 7 and Elise genotypes, respectively) and the highest $\mathrm{N}$ level of $60 \mathrm{~kg} \mathrm{~N} / \mathrm{fad}$ under the environmental conditions prevailing in the surrounding media as well as their considerable ability to convert a greater portion of metabolites to their sinks, viz : fruiting forms or fibre units.

\section{REFERENCES}

A.O.A.C. (1980). Association of Official Agriculture Chemists. Official Methods of Analysis, $13^{\text {th }}$ ed, Washington, D.C.

Ash-Shormillesy, Salwa, M.E.I. (2001). Effect of seeding rate and fertilization on yield and quality of flax. Ph.D. Thesis, Fac. of Agric., Zagazig Univ., Egypt.

Das, M.N. and N.C. Giri (1986). Design and Analysis of Experiments. $2^{\text {nd }}$ Eddition, John Wiley and Sons. Inc., New York, USA.

Duncan, D.B. (1955). Multiple range and multiple F-tests, Biometrics, 11:1-42. 
OMAR ET AL 
El-Azzouni, A.M.; E.A. Moawed and S.M. Salama (2003). Effect of seeding rate, potassium fertilizer on some genotypes of flax (Linum usitatissimum L.). J. Agric. Sci. Mansoura Univ., 28(8) : 5887-5902.

El-Deeb, T.A.E.; N.A. Mohamed and Eman, A.F. El-Kady (2006). Effect of seeding rates, planting methods and phosphorus fertilizer sources on yield and its components of Sakha 2 flax cultivar. Zagazig. J. Agric. Res., 33(1) : $27-45$.

El-Gazzar, A.A.M. (1990). Effect of some cultural treatments on flax yield and quality. M.Sc. Thesis, Fac. of Agric., Kafr El-Sheikh, Tanta Univ., Egypt.

El-Gazzar, A.A.M. (2005). Effect of seeding rates, phosphorus and potassium fertilizers on growth, yield and quality of flax. J. Agric. Sci. Mansoura Univ., 30 (12) : 7239-7253.

El-Gazzar, A.A.M. and Eman A. El-Kady (2005). Effect of some growth regulators and nitrogen levels on flax yield and its quality. J. Agric. Res. Tanta Univ., 31(4A) : 630-646.

El-Gazzar, A.A.M. and M.E.A. Kineber (2002). Response of flax yield and quality to nitrogen fertilizer and its sources. J. Agric. Res. Tanta Univ., 28(1) : 93 - 104.

El-Gazzar, A.A.M. and T.A. Abou-Zaied (2001). Effect of seeding rate and nitrogen levels on yield and quality of some flax cultivars. J. Agric. Res. Tanta Univ., 27(4) : 607-619.

El-Sabbagh, A.A.; S.A. Abd El-Hafez and El-Sh. T. Abou-Ahmed (1998). Response of some flax cultivars to water stress. Menufiya J. Agric. Res., 23(5) : 13671376.

El-Sweify, Amna, H.H.; E.A.F. El-Kady and S.M. Gafar (1996). The influence of harvesting time and row spacing on the quantity, quality and chemical composition of some flax cultivars. J. Agric. Sci. Mansoura Univ., 21(10) : 2421 $-2428$.

Esmail, S.E. and M.R. Morsy (1994). Flax plants performance as influenced by seeding rate and harvesting date and their response curves. Menufiya J. Agric. Res., 19(5) : 2243 - 2255.

Ghanem, S.A.I. (1990). The influence of $\mathrm{N}$ fertilization and harvesting dates on oil, fibre yields and their contributing characters of flax. Zagazig J. Agric. Res., 17(3A) : $575-587$.

Kineber, M.E.A. (2003). Flax plants performance as influenced by planting methods and seeding rate. J. Agric. Res. Tanta Univ., 29(1): $64-73$.

Kineber, M.E.A. ; A.A.E. Mohamed and E.A.F. El-Kady (1997). Influence of planting method and seeding rate on yield and its components of some flax genotypes. J. Agric. Res. Tanta Univ., 23(3) : $289-299$.

Kineber, M.E.A. ; S.Z. Zedan and E.A.F. El-Kady (1998). Response of flax variety Giza8 to different levels of nitrogen and foliar application of some microelements. J. Agric. Sci. Mansoura Univ., 23 (8) : 2591 - 2599. 
Moustafa, S.H.; M.E.A. Kineber and A.A.E. Mohamed (2003). Effect of inoculation with phosphorine and nitrogen on flax yield and some anatomical characters under different nitrogen levels. J. Agric. Sci. Mansoura Univ., 28(6) : 43074323.

Zedan, S.Z.; M.E.A. Kineber and S.H. Moustafa (1997). Response of flax to potassium and nitrogen fertilization under sandy soil conditions. Egypt J. Agric. Res., 27(2) : 729 - 743.

تأثير معدل التقاوي والتسميد النيتروجيني على المحصول في الكتان عبد الرحمن السيد أحمد عمر - سلوى محمد اليماني إبراهيم الثرملسى قسم المحاصيل - كلية الزراعة - جامعة الزقازيق.

أجريت هذه الدراسة فى محطة البحوث الزراعية (موقع غزالة) - كلية الزراعة - جامعة الزقازيق - جمهورية

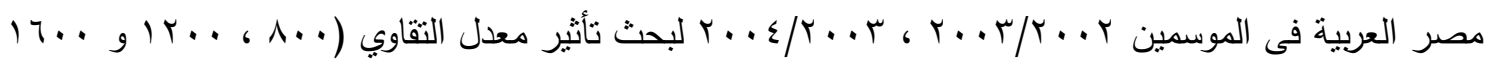

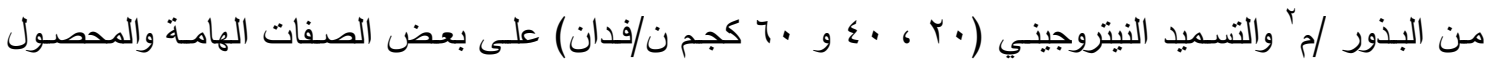
النهائي فى وحدة المساحة لصنفي الكتان إليز (Elise) وجيزة > (Giza 7). تم استخدام نظام القطع الثقية من بن الدرجة الثانية ذو الثلاث مكررات فى التصميم التجريبي ، حيث شغلت القطع الرئيسية بالصنفين ، ووزعت معدلات التقاوي الثلات فى القطع الثقية الأولى ، بينما كانت مستويات التسميد النيتروجيني الثلات فى القطع الثقية الثانية

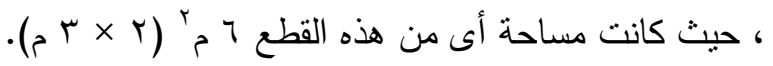

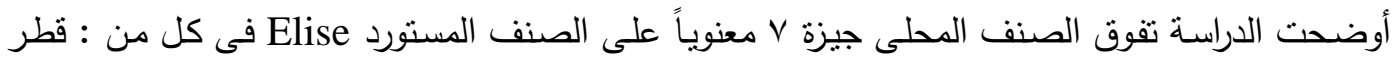

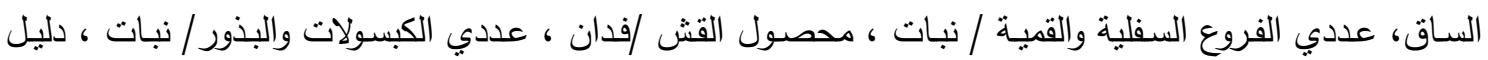

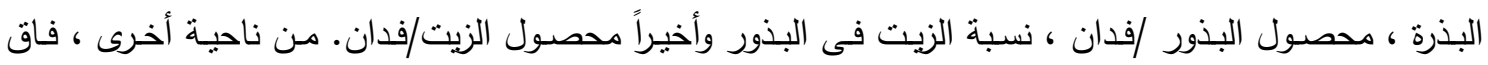
الصنف Elise الصنف جيزة V فى : ارتفاع النبات ، الطول الفعال ، نسبة الألياف ومحصول الألياف / فدان. ظهرت هذه الاختلافات الصنفية بوضوح فى كلا الموسمين وفى التحليل المشترك لهما.

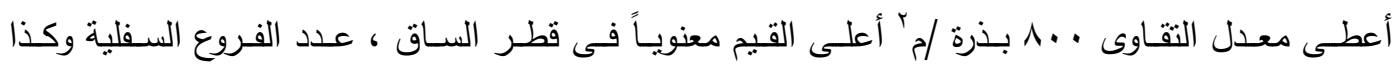
القمية/نبات ، عدد الكبسولات وبالمثل البذور /نبات ، دليل البذرة وأخيراً نسبة الزيت فى البذور • وعلاوة على ذلى ذلك

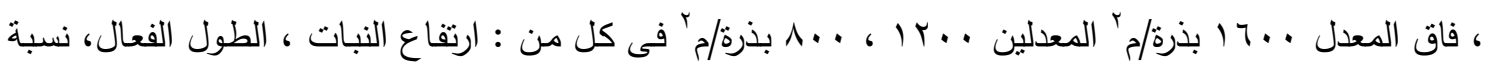


الألياف وأخيراً الدحصول النهائي فى وحدة المساحة من : القش ، الألياف ، البذور والزيت. ظهرت هذه الاختلافات

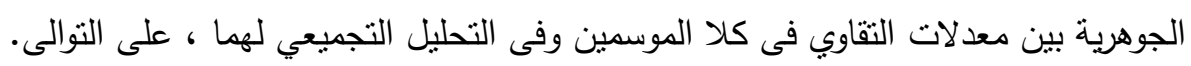

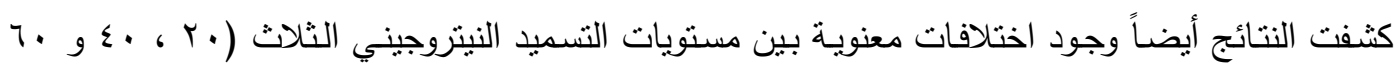
كجم ن/فدان) ، حيث أعطى المستوى • r كجم ن/فدان زيادة معنوية فى نسبة الألياف ومحتوى الزيت فى البذرة.

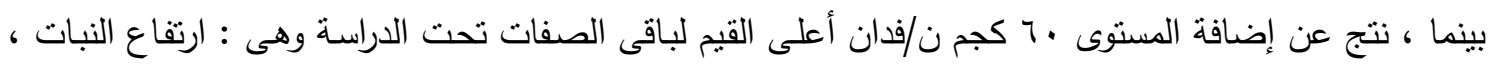

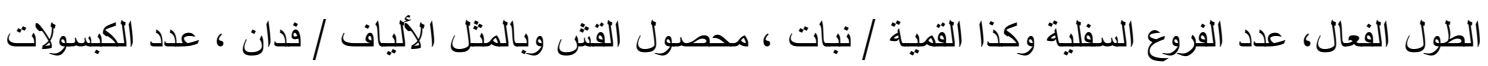

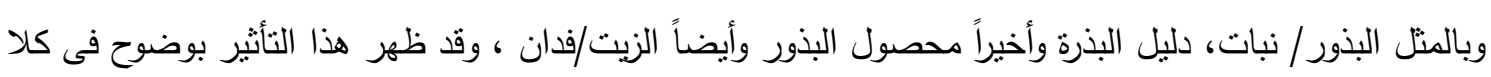
التجربتين وفى التحليل المشترك لهما.

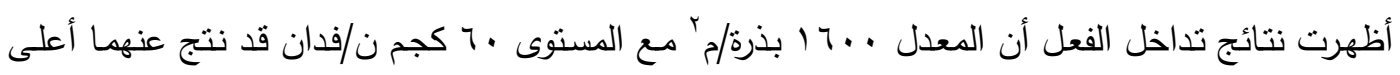
القيم في كل من : ارتفاع النبات ، نسبة الألياف ومحصول الألياف/فدان (للصنف المستورد : Elise) ، عدد الفروع

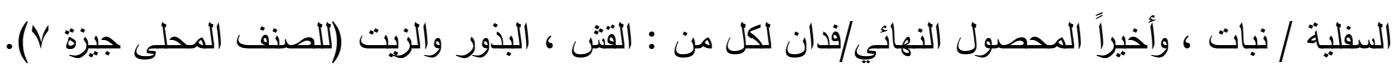
التوصية :

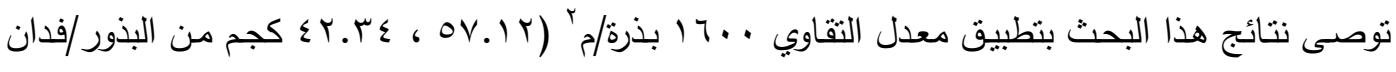

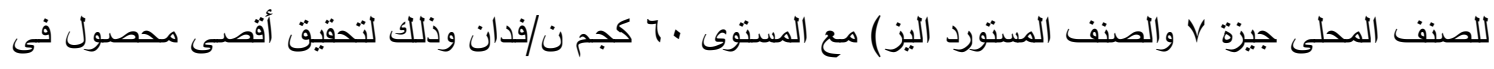
وحدة المساحة من الزيت والألياف تحت ظروف مزرعـة كلية الزراعـة - جامعة الزقازيق - محافظة الثـرقية جمهورية مصر العربية. 